\title{
Differential Simultaneous Liver and Kidney Transplant Benefit Based on Severity of Liver Damage at the Time of Transplantation
}

\author{
Shahid Habib ${ }^{\mathrm{a}, \mathrm{g}}$, Khalid Khan ${ }^{\mathrm{b}}$, Chiu-Hsieh Hsuc ${ }^{\mathrm{c}}$, Edward Meister ${ }^{\mathrm{d}, \mathrm{f}}$, \\ Abbas Rana ${ }^{\mathrm{e}}$, Thomas Boyer ${ }^{\mathrm{d}}$
}

\begin{abstract}
Background: We evaluated the concept of whether liver failure patients with a superimposed kidney injury receiving a simultaneous liver and kidney transplant (SLKT) have similar outcomes compared to patients with liver failure without a kidney injury receiving a liver transplantation (LT) alone.
\end{abstract}

Methods: Using data from the United Network of Organ Sharing (UNOS) database, patients were divided into five groups based on pre-transplant model for end-stage liver disease (MELD) scores and categorized as not having (serum creatinine $(\mathrm{sCr}) \leq 1.5 \mathrm{mg} / \mathrm{dL}$ ) or having $(\mathrm{sCr}>1.5 \mathrm{mg} / \mathrm{dL})$ renal dysfunction. Of 30,958 patients undergoing LT, 14,679 (47.5\%) had renal dysfunction, and of those, $5,084(16.4 \%)$ had dialysis.

Results: Survival in those (liver failure with renal dysfunction) receiving SLKT was significantly worse $(\mathrm{P}<0.001)$ as compared to those with $\mathrm{sCr}<1.5 \mathrm{mg} / \mathrm{dL}$ (liver failure only). The highest mortality rate observed was $21 \%$ in the $36+$ MELD group with renal dysfunction with or without SLKT. In high MELD recipients (MELD > 30) with renal dysfunction, presence of renal dysfunction affects the outcome and SLKT does not improve survival. In low MELD recipients (16 - 20), presence of renal dysfunction at the time of transplantation does affect post-transplant survival, but survival is improved with SLKT.

Manuscript accepted for publication March 01, 2017

aLiver Institute, PLLC, 2830 North Swan Road, Suite 180, Tucson, AZ 85712, USA

${ }^{\text {b} T r a n s p l a n t ~ I n s t i t u t e, ~ M e d S t a r ~ G e o r g e t o w n ~ U n i v e r s i t y ~ H o s p i t a l, ~} 3800$ Reservoir Rd, Main, Washington, DC 20007, USA

${ }^{\mathrm{c}}$ Division of Epidemiology and Biostatistics, Mel and Enid Zuckerman College of Public Health, The University of Arizona, Tucson, AZ 85742, USA

dDepartment of Medicine and Surgery, Divisions of Gastroenterology, Hepatology and Liver Transplantation, Liver Research Institute, College of Medicine, University of Arizona, Tucson, AZ 85742, USA

eDepartment of Surgery, Division of Transplantation, Baylor College of Medicine, Houston, TX 77030, USA

fDeceased (biostatistician).

gCorresponding Author: Shahid Habib, Liver Institute, PLLC, 2830 N Swan

Road, Suite 180, Tucson, AZ 85712, USA.

Email: shabib@liverinstitutepllc.org

doi: https://doi.org/10.14740/gr803w
Conclusions: SLKT improved 1-year survival only in low MELD (16 - 20) recipients but not in other groups. Performance of SLKT should be limited to patients where a benefit in survival and post-transplant outcomes can be demonstrated.

Keywords: MELD; Liver transplantation; Patient survival; Graft survival; Kidney dysfunction; Simultaneous liver and kidney transplantation

\section{Introduction}

Deceased donor organs for liver transplantation (LT) are prioritized to patients with the greatest immediate risk for death on the waiting-list utilizing the model for end-stage liver disease (MELD) scoring system. The newest guidelines from the United Network of Organ Sharing (UNOS) have taken this one step further in the United States with the "share 35" allocation system where adult patients with a MELD score of $\geq 35$ benefit from regionally and nationally available organs in comparison to others on the waiting-list. This policy resulted in an increase in the number of liver transplant allograft recipients with high $\operatorname{MELD}(\geq 30)$ scores by $10 \%$ from 2012 to 2016 [1]. As serum creatinine ( $\mathrm{sCr}$ ) level is an important component in the MELD calculation, it is expected that a proportion of such patients may require a kidney allograft in addition to liver transplant because of associated renal injury.

Renal dysfunction is heavily weighted in MELD calculation as it has strong impact on survival before transplantation [2]. The prevalence of kidney dysfunction $(\mathrm{sCr}>1.5 \mathrm{mg} / \mathrm{dL})$ at the time of transplantation is increasing since the adoption of the MELD allocation model. For example, $26 \%$ of transplanted patients in $2002 \mathrm{had}$ a sCr of $>1.5 \mathrm{mg} / \mathrm{dL}$ and that number has increased to $33 \%$ in 2005 and continues to rise $[3,4]$. The impact of the presence of renal insufficiency at the time of transplantation on post-transplantation survival has been the focus of several studies. For patients transplanted with the same MELD score, the level of sCr is inversely associated with survival within certain defined MELD categories [5]. A pre-transplantation $\mathrm{sCr}$ of $>2 \mathrm{mg} / \mathrm{dL}$ is associated with $58 \%$ greater risk of post-transplant death compared to recipients with a creatinine of $<1 \mathrm{mg} / \mathrm{dL}$ [6].

Not only does pre-transplant renal dysfunction appear to 
increase the risk of dying post-transplant but it also increases the number of simultaneous liver and kidney transplants (SLKT) [7], which are performed with the concept and intention to overcome dual organ (liver and kidney) failure and restore the post-transplantation survival equal to those liver allograft recipients without renal dysfunction. This concept has not been validated. Though, several studies have looked into timing of kidney transplantation (KT) in such patients. The benefits of SLKT vs. delayed KT are a matter of debate [8-14]. Martin et al utilizing UNOS data found that after adjustments for potential confounding demographic and clinical variables, there was significantly lower mortality in those receiving LT alone as compared to SLKT. However, SLKT improved mortality and graft survival compared to LT followed by KT [6]. In another study of those with end-stage liver disease (ESLD) and kidney dysfunction not on dialysis, liver graft survival of patients who underwent SLKT was superior to those of patients who underwent LT alone [15]. Lastly, a recent Markov model suggested that irrespective of MELD score, SLKT gave better survival compared to LT followed by KT in patients on renal replacement therapy (RRT) preceding LT [16]. However, the model failed to consider that many patients on RRT for $<$ 30 days have recovery of renal function. The model also failed to consider the impact of the loss of organs for SLKT on the survival of those awaiting KT alone [17].

Natural history of acute kidney dysfunction (AKD) in advanced liver disease patients is not well studied. Several factors have been implicated in the pathogenesis of AKD such as portal hypertension, spontaneous bacterial peritonitis (SBP) and use of nephrotoxic agents. Little is known about factors predictive of AKD outcome in the setting of advanced liver disease. In clinical practice, it is observed that a good proportion of patients recover baseline renal functions with supportive treatment including short-term dialysis before and after LT. Nonetheless, several questions remain unanswered such as the magnitude and timing of recovery, impact of severity of underlying liver disease and severity of portal hypertension, and impact of systemic diseases such as diabetes. Sharma et al studied the effect of renal dysfunction at the time of transplantation on post-transplantation outcome and it was determined that survival varied depending upon severity of liver disease at the time of transplantation [5]. This could explain the reasons for inconsistent benefits of KT in liver disease patients. Moreover, any MELD point could be reflective of three possibilities: 1) liver dysfunction alone, 2) renal dysfunction alone, and 3) combined liver and kidney dysfunction. The outcome of each MELD point could be different based on the above-mentioned possibilities.

We evaluated the concept of whether liver failure patients with superimposed kidney injury receiving SLKT have similar outcomes compared to patients with liver failure without kidney injury receiving LT alone. This will help to better define the timing of LT. We have examined how MELD score and the presence or absence of renal dysfunction (MELD score reflective of 1) liver disease alone and 2) combined liver and kidney dysfunction) at the time of transplantation influenced post-transplant survival both in those receiving LT alone or SLKT. Using the UNOS database, we analyzed the differences in survival in patients with and without renal dysfunction at the time of transplantation as well as the influence of MELD and SLKT on survival in these two groups of patients. The results of this analysis are the subject of this report.

\section{Patients and Methods}

\section{Study population}

We examined the Organ Procurement and Transplant Network (OPTN)/UNOS, Standard Transplant Analysis and Research (STAR) database information for this study. The OPTN database is a clinical data resource that includes demographics on all wait-list candidates in the US as submitted by individual transplant centers, and STAR files are provided as de-identified data. We observed the University of Arizona Review Board guidelines for analysis of de-identified data for this study. We evaluated data inclusive of all adults aged $\geq 18$ years receiving transplants since May 2002. We selected this time frame because the MELD scoring system was implemented in February 2002. Exclusion criteria included: patients receiving transplantation with MELD scores $<15$, those that were given exception points or had a living donor, and those lost to follow-up. Patients were divided into five groups based on the pre-transplant MELD score: 16 - 20, 21 - 25, 26 - 30, 31 35 , and 36 and above $(+)$. The ranges of the MELD groupings were selected after analysis of smaller and larger groups and were considered to be the best sizes to demonstrate betweengroup changes. Each group was also stratified based on the level of renal dysfunction and a sCr cut-off of $1.5 \mathrm{mg} / \mathrm{dL}$ was used. This stratification was based on the guidelines of International Ascites Club's definition of renal failure in the setting of cirrhosis $[18,19]$. Patients who had $\mathrm{sCr} \leq 1.5 \mathrm{mg} / \mathrm{dL}$ and were on dialysis at transplantation were included in the renal dysfunction group $(\mathrm{sCr}>1.5 \mathrm{mg} / \mathrm{dL})$. Based on presence or absence of renal dysfunction, each MELD group was stratified into: 1) liver MELD ( $\mathrm{sCr} \leq 1.5 \mathrm{mg} / \mathrm{dL}$, reflecting liver failure only), receiving LT alone, 2) liver-kidney MELD ( $\mathrm{sCr}>1.5$ $\mathrm{mg} / \mathrm{dL}$, reflecting combined liver and kidney failure), receiving LT alone, and 3) liver-kidney MELD ( $\mathrm{sCr}>1.5 \mathrm{mg} / \mathrm{dL})$, receiving an SLKT.

\section{Statistical analysis}

We performed descriptive statistics to determine the patient and donor characteristics. For continuous variables, Student's $t$-test and an analysis of variance (ANOVA) were utilized. For categorical variables, we used Fisher's exact test and the Chisquare test with Yates continuity correction if applicable. Pearson's correlation and ANOVA were used to analyze trends and differences between the groups. Cumulative survival was determined using a Kaplan-Meier analysis, and the groups were compared using a log-rank test. Factors predictive of longterm survival were determined via Cox proportional hazards modeling, and the results are given in terms of hazard ratios (HRs). Only the variables found to be significant on univariate analysis were included for Cox proportional hazard modeling. 
Table 1. Clinical Characteristics at Transplantation

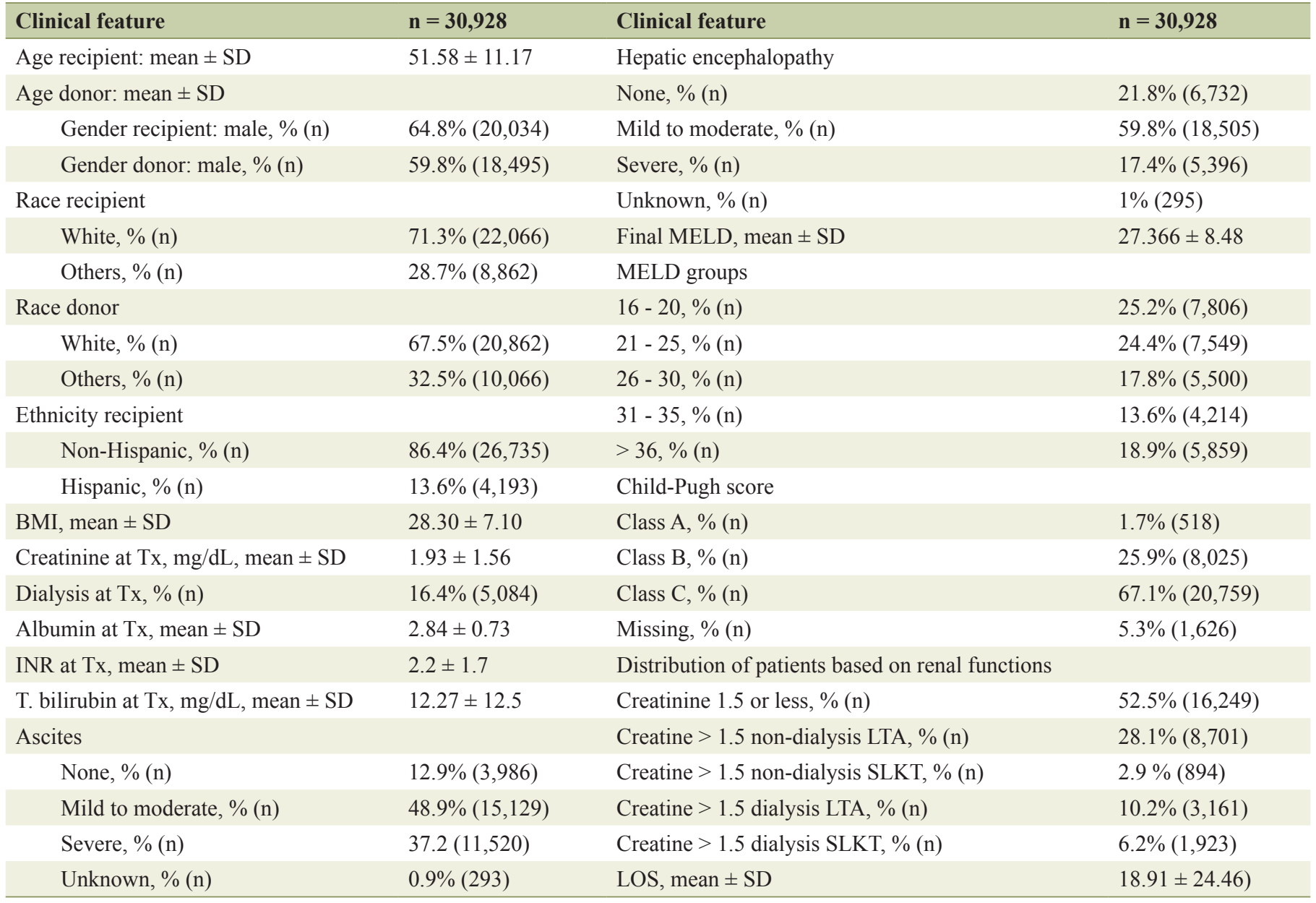

SD: standard deviation; n: sample number; MELD: model for end-stage liver disease; BMI: body mass index; LTA: liver transplantation alone; SLKT: simultaneous liver and kidney transplantation; LOS: length of stay.

All analyses were performed with the Statistical Package for the Social Sciences (SPSS 22, IBM Corp., Armonk, NY, USA) and Statistical Analysis System (SAS ${ }^{\circledR}$, SAS Institute Inc. Cary, NC, USA). A P-value $\leq 0.05$ was considered to indicate statistical significance.

\section{Results}

Data were analyzed on 30,958 patients undergoing LT who met inclusion and exclusion criteria. Clinical features of the study cohort are shown in Table 1 . The mean age of the cohort was $51.58 \pm 11.18$ years, of which 20,034 (64.8\%) were male. Caucasian race represented $71 \%$ of the study cohort, African American comprised 11\% and 4,193 (13.6\%) were Hispanic. At the time of transplantation, the mean MELD score was $27.37 \pm 8.49$, and total bilirubin $(\mathrm{mg} / \mathrm{dL}), \mathrm{INR}$, and creatinine $(\mathrm{mg} / \mathrm{dL})$ were $12.27 \pm 12.5,2.2 \pm 1.7$, and $1.93 \pm 1.56$, respectively. Of the total cohort, $14,679(47.5 \%)$ had a $\mathrm{sCr}>1.5$ $\mathrm{mg} / \mathrm{dL}$ at time of transplantation. Of the latter group, 5,084 (16.4\%) had received RRT (Table 1). The distributions of pa- tients in different MELD groups, by renal dysfunction (creatinine $>1.5 \mathrm{mg} / \mathrm{dL}$ ), and those that received a LT alone versus SLKT, are shown in Table 2.

\section{MELD score and kidney dysfunction}

We analyzed the bivariate correlation between MELD score and $\mathrm{sCr}$ at transplantation (continuous variables using Pearson correlation test). Mean $\mathrm{sCr}$ at transplantation rose progressively from low MELD to high MELD groups with a statistically significant positive correlation $(\mathrm{R}=0.44, \mathrm{P}$ $=0.0001$ ) (Fig. 1). Overall, at the time of transplantation, $47.5 \%$ of study cohort had MELD score reflective of combined liver and kidney failure (liver-kidney MELD). In analyzing the trends with increasing MELD scores, there was a notable increase in the proportion of patients with renal impairment in successive MELD groups which was significant between all of the groups (Fig. 2). Reflecting this increase in renal dysfunction, there also was a proportional increase in the number of patients receiving SLKT as the MELD score 
Table 2. Distribution of Patients Based on Serum Creatinine Status at Liver Transplantation

\begin{tabular}{llllll}
\hline MELD group & Creatinine $\leq \mathbf{1 . 5} \mathbf{~ m g / d L}$ & Creatinine $>\mathbf{1 . 5} \mathbf{~ m g / d L}$ & LT alone & SLK & Death at 1 year \\
\hline $16-20(\%)$ & $6,584(21.3 \%)$ & $1,222(4.0 \%)$ & $7,594(24.6 \%)$ & $212(0.7 \%)$ & $664(2.2 \%)$ \\
$21-25(\%)$ & $4,952(16.0 \%)$ & $2,597(8.4 \%)$ & $6,793(22.0 \%)$ & $756(2.4 \%)$ & $847(2.7 \%)$ \\
$26-30(\%)$ & $2,832(9.2 \%)$ & $2,668(8.6 \%)$ & $4,902(15.9 \%)$ & $598(1.9 \%)$ & $731(2.4 \%)$ \\
$31-35(\%)$ & $1,289(4.2 \%)$ & $2,925(9.5 \%)$ & $3,695(12.0 \%)$ & $519(1.7 \%)$ & $632(2.0 \%)$ \\
$35+(\%)$ & $592(1.9 \%)$ & $5,267(17.0 \%)$ & $5,127(16.6 \%)$ & $732(2.4 \%)$ & $1,091(3.5 \%)$ \\
Total (\%) & $16,249(52.5 \%)$ & $14,679(47.5 \%)$ & $28,111(90.9 \%)$ & $2,817(9.1 \%)$ & $3,965(12.8 \%)$ \\
\hline
\end{tabular}

MELD: model for end-stage liver disease; LT: liver transplant; SLKT: simultaneous liver and kidney transplant.

increased (Fig. 3).

\section{Survival analysis}

In each of the MELD groups, survival at 1 year of patients with $\mathrm{sCr} \leq 1.5 \mathrm{mg} / \mathrm{dL}$ (liver MELD) receiving LT alone was significantly better compared to those with $\mathrm{sCr}>1.5 \mathrm{mg} / \mathrm{dL}$ (liver-kidney MELD) (Fig. 4). Difference in survival between the groups became greater with increasing MELD score, i.e. at 3\% difference with MELD of 16 - 20 vs. a $6 \%$ difference with MELD of 36 - 40. When comparing the group with $\mathrm{sCr} \leq$ $1.5 \mathrm{mg} / \mathrm{dL}$ (liver MELD) and receiving LT alone to those with $\mathrm{sCr}>1.5 \mathrm{mg} / \mathrm{dL}$ (liver-kidney MELD) and receiving SLKT, patient survival at 1 year was comparable in the lowest MELD group: 16 - $20(\mathrm{P}=0.8)$. In higher MELD groups, patients without renal dysfunction (liver MELD) had improved survival compared to those with renal dysfunction (liver-kidney MELD) irrespective of whether or not they had LT alone or SLKT (Fig. 4).

We performed Cox regression model analysis to identify the impact of kidney dysfunction during the transplantation phase and at 1 year post-transplantation. The following variables were significantly associated with higher mortality during transplantation phase: $\mathrm{sCr}>1.5$ (HR: 1.710 (1.244 - 2.349); $\mathrm{P}<0.001)$ and dialysis at transplantation (HR: 1.837 (1.304 - 2.589); $\mathrm{P}<0.001)$. Male gender was associated with lower mortality risk (HR: 0.681 (0.529 - 0.879); $\mathrm{P}<0.01)$. Factors associated with long-term survival at 1 year after excluding patients who died during transplantation phase were recipient and donor age, recipient BMI, recipient and donor non-white race, non-Hispanic ethnicity, $\mathrm{sCr}>1.5 \mathrm{mg} / \mathrm{dL}, \mathrm{sCr}>1.5 \mathrm{mg} /$

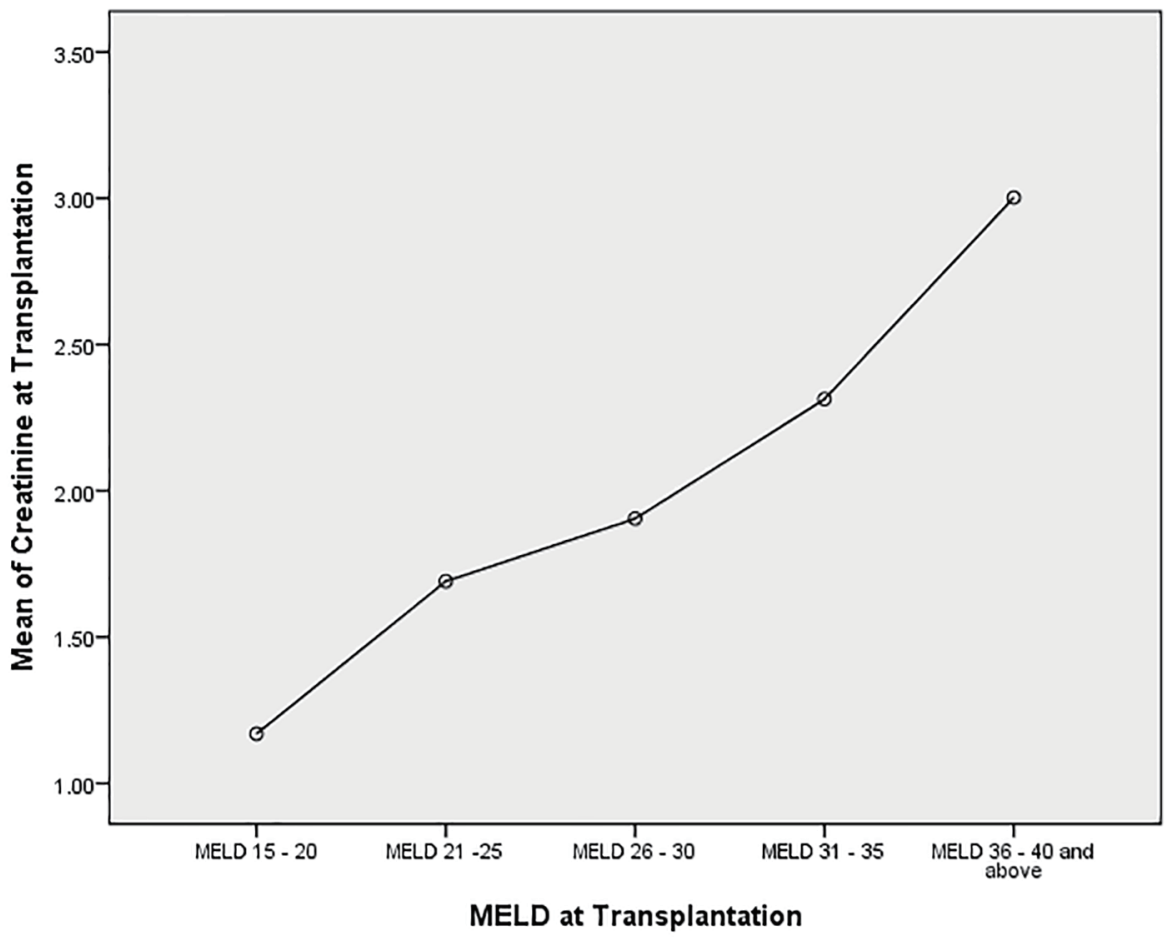

Figure 1. Correlation between serum creatinine and MELD score categories at the time of transplantation. Analysis of variance (ANOVA): mean serum creatinine in each MELD group $(P=0.0001)$. A linear correlation between increasing MELD score and serum creatinine (Pearson correlation coefficient 0.04, $P=0.0001$ ). 


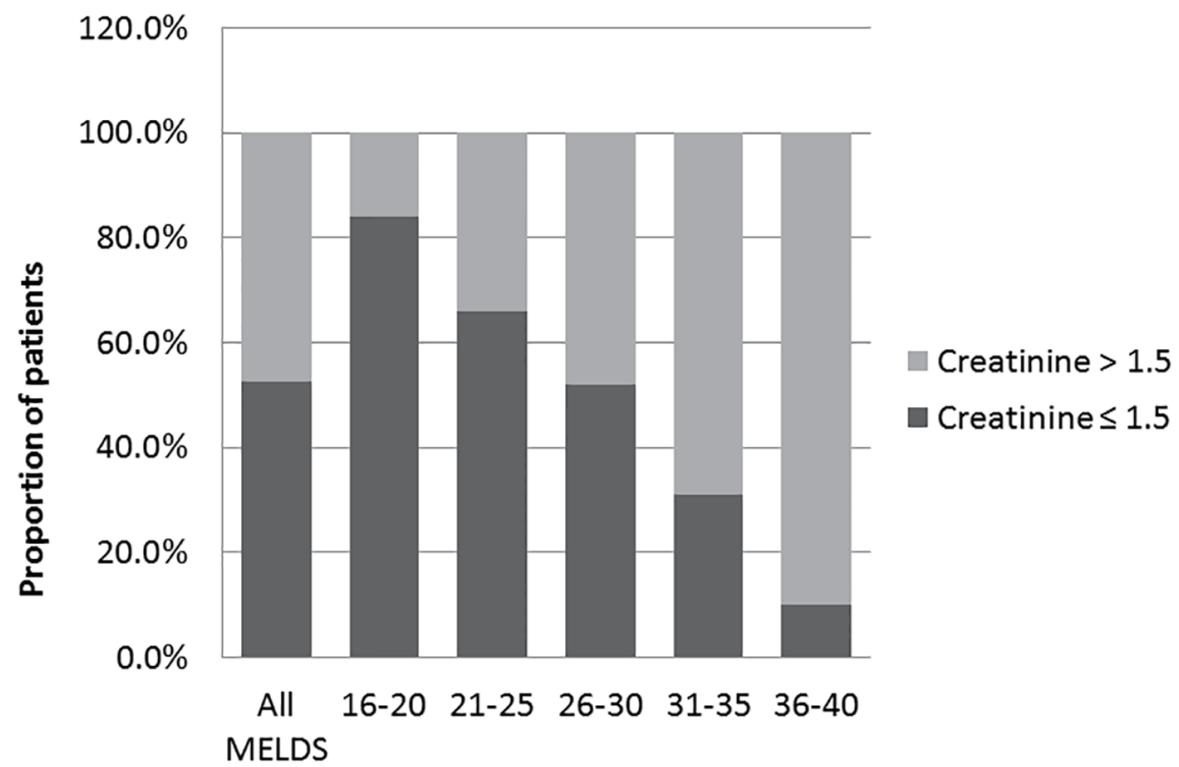

MELD score at transplantation

Figure 2. Prevalence of renal dysfunctions at the time of transplantation. Prevalence of patients with renal dysfunctions creatinine > 1.5 in overall study cohort and in each MELD group. A trend of increasing prevalence from low to high MELD groups (Pearson correlation $\mathrm{R}$ 0.46: $\mathrm{P}=0.0001$ (two-tailed)). One-way analysis of variance was used to determine the differences among the groups. All the groups are statistically significant from each other $(P=0.001)$.

$\mathrm{dL}$ and LT only, $\mathrm{sCr}>1.5 \mathrm{mg} / \mathrm{dL}$ and SLKT, dialysis at transplantation and MELD score (Table 3).

We also analyzed distribution of deaths based on $\mathrm{sCr}$ status both during the transplantation phase and at 1 year post-trans- plantation (Table 4). Total number of deaths within first year transplantation for the entire cohort was 3,965 (12.8\%). The largest proportions $(28 \%)$ of deaths were in the $36+$ MELD groups (Fig. 5a). We analyzed the distribution of deaths based

$\square$ LA $\square$ SLK

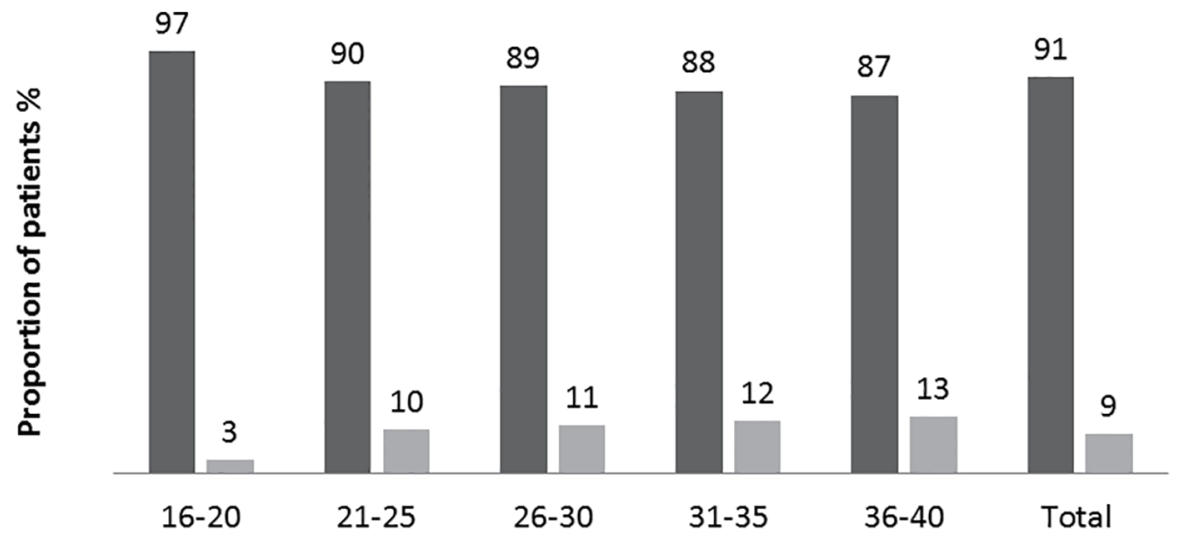

MELD score at transplantation

Figure 3. Distribution of patients receiving transplantation base on MELD categories. Proportion of patients receiving simultaneous liver kidney (SLK) or liver alone (LA) transplant in each MELD group and in overall study population. A trend of increasing prevalence from low to high MELD groups (Pearson correlation $R$ 0.11: $P=0.00001$ (two-tailed)). Analysis of variance was used to determine the differences among the groups. High MELD group (MELD $36-40)$ is statistically significantly different $(P \leq 0.05)$ compared to other groups except MELD group $31-35(P=1.0)$. 


\section{Group 1: Creatinine $\leq 1.5 \quad$ Group 2: Creatinine > 1.5 LA}

Group 3: Creatinine > 1.5 SLK

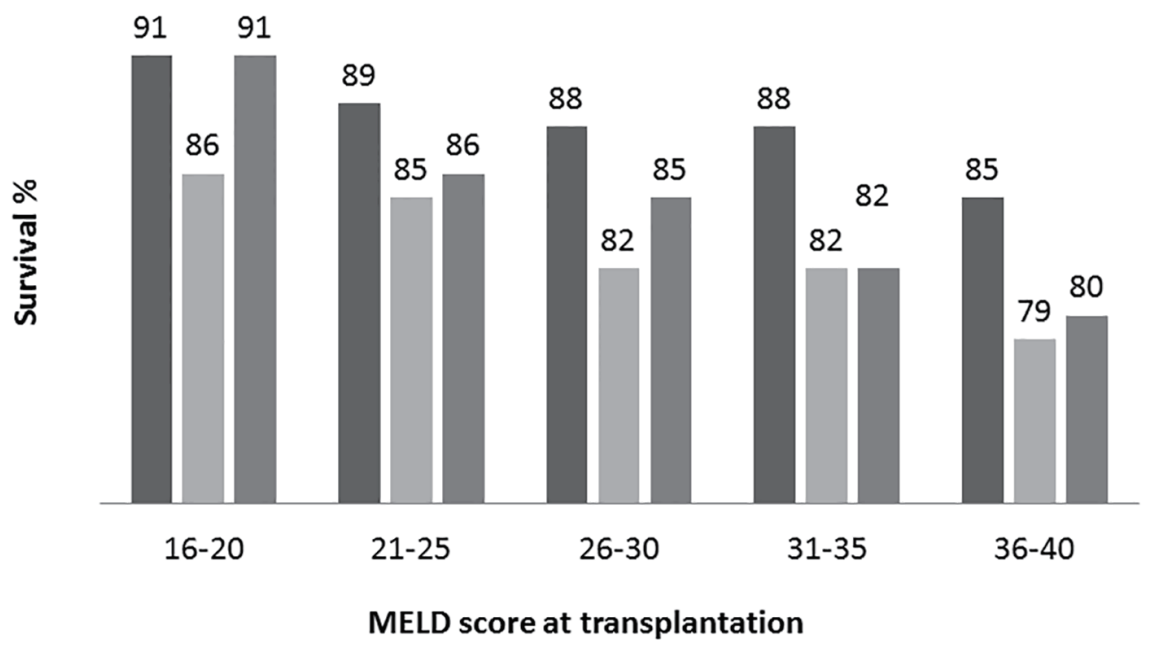

Figure 4. Kaplan-Meier patient survival analysis at 1 year based on MELD category. In each MELD category survival was compared among three transplant recipient groups (group 1: creatinine $<1.5$ receiving liver alone, group 2: creatinine $>1.5$ receiving liver alone and group 3: creatinine $>1.5$ receiving simultaneous liver kidney. Log rank test $P$ values (statistically significant $<0.05$ ) are: 16 - 20 ( 1 vs. 2 ); 21 - 25 ( 1 vs. 2 and 1 vs. 3); 26 - 30 (1 vs. 2); 31 - 35 (1 vs. 2 and 1 vs. 3); 36 - 40 ( 1 vs. 2 and 1 vs. 3 ).

on renal dysfunction (liver MELD vs. liver-kidney MELD) in each MELD group. A greater percentage of the total deaths occurred in recipients with renal dysfunction (liver-kidney MELD) at transplantation compared to those without renal dysfunction (liver MELD): 2,414 (61\%) vs. 1,551 (39\%). With increasing MELD score, the proportion of deaths of patients with renal dysfunction at the time of transplant increased from $21 \%$ of those dying with MELD of $16-20$ to $92 \%$ of those dying in the $36+$ MELD group. Based on this analysis, one out of five patients with a MELD score $>36$ and $\mathrm{sCr}>1.5 \mathrm{mg} / \mathrm{dL}$ had died within 1 year of transplantation (Fig. 5a, b).

\section{Discussion}

MELD was developed to predict survival following creation of a transjugular intrahepatic portosystemic shunt (TIPS) and was then used to predict survival for patients waiting for liver transplant. It is now becoming increasingly apparent that severity of liver disease based on MELD score at the time of transplant is also predictive of post-transplant survival. Development of renal disease is an important milestone in the course of cirrhotic patients. In the absence of recovery from renal dysfunction, it has been shown to be associated with very high mortality without transplantation; therefore, it carries significant weight in the MELD calculation [20]. The causes of renal insufficiency in this patient population include acute kidney injury (AKI) due to decreases in plasma volume, infection and hepatorenal syndrome and chronic renal disease due to diseases like diabetes mellitus [21]. Use of volume expansion, antibiotics and vasopressors such as terlipressin has the potential for reversing the AKI preceding transplant $[22,23]$. Failure to improve the re- nal insufficiency means many patients undergoing LT will also have significant renal insufficiency. In this report, we demonstrate the impact of renal insufficiency on survival following LT over a range of MELD scores. Irrespective of the MELD score, survival was significantly worse in those with renal insufficiency as compared to those without renal insufficiency (Fig. 4). The impact of renal insufficiency on survival was greatest in those with a high MELD score. For example, $90 \%$ of patients transplanted with a MELD of 36 - 40 had elevated $\mathrm{sCr}$ and of those who died within 1 year of transplant, $92 \%$ had renal insufficiency. In contrast, only $16 \%$ of those with a MELD of $16-20$ had renal insufficiency and those with $\mathrm{sCr}>1.5$ accounted for only $21 \%$ of the deaths in this group (Figs. 2 and 5 b). Thus, in the high MELD patients, renal insufficiency is increasingly common and associated with high 1-year mortality. The adoption of the "share 35" rule will only increase the number of patients in this high risk group. The findings in this analysis are supported by previous reports that patients with renal insufficiency at the time of transplant have worse outcomes [5, $6]$. However, the combined effect of MELD and renal insufficiency was not part of the latter reports analyses.

Not surprisingly, the number of SLKT has increased since the adoption of MELD which has impacted the availability of donor kidneys for patients with ESRD $[6,14,15]$. Reports using modeling or analysis of the UNOS database have suggested that SLKT is better than delayed KT for both graft and patient survival $[6,14,15]$. Furthermore, the addition of the liver graft to the kidney in sensitized individuals has been shown to reduce the possibility of acute rejection suggesting that for patients with a high level of donor-specific antibody simultaneous transplantation may be advantageous [24]. However, when survival of SLKT patients is compared to LT alone patients the data are 
Table 3. Cox Proportional Hazard Analysis

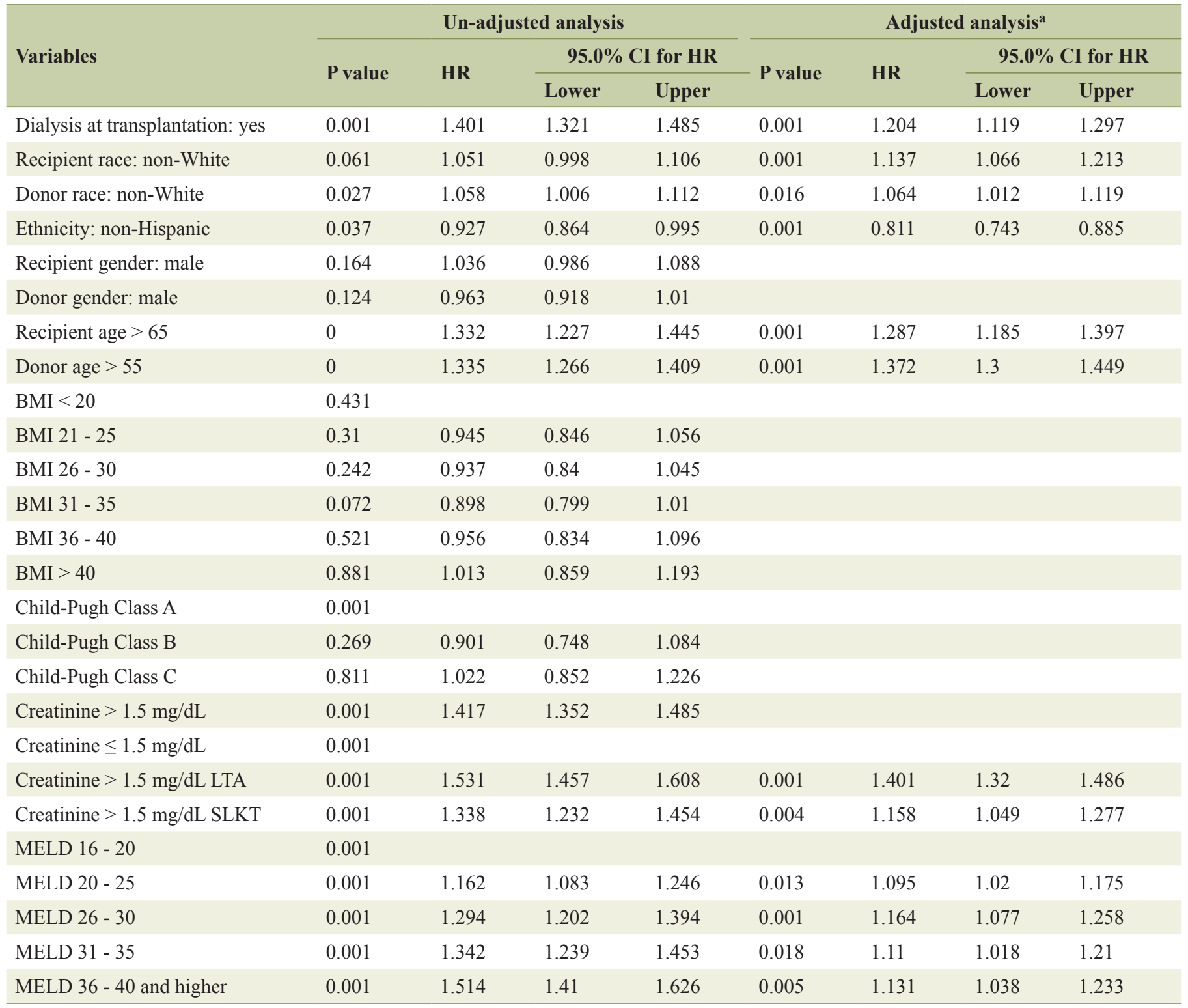

HR: hazard ratio; Cl: confidence interval; MELD: model for end-stage liver disease; BMI: body mass index; LTA: liver transplantation alone; SLKT: simultaneous liver and kidney transplantation. ${ }^{a}$ Adjusted for patient's ascites grade, albumin, bilirubin, Cr, INR, MELD, dialysis, gender, race and age, donor's age and gender, and type of transplant.

Table 4. Death by $\operatorname{Cr}(>1.5$ vs. $\leq 1.5)$ and MELD Score $(\geq 30$ vs. $<30)$

\begin{tabular}{|c|c|c|c|c|}
\hline \multirow{2}{*}{ Group } & \multicolumn{2}{|c|}{ Transplant phase } & \multicolumn{2}{|c|}{ At 1-year post-transplantation ${ }^{b}$} \\
\hline & Number of deaths $(\%)$ & Adjusted P-value ${ }^{a}$ & Number of deaths (\%) & Adjusted P-value ${ }^{a}$ \\
\hline $\mathrm{Cr} \leq 1.5$ and $\mathrm{MELD}<30$ & $85(0.62 \%)$ & $<0.001$ & $2,510(18.34 \%)$ & $<0.0001$ \\
\hline $\mathrm{Cr} \leq 1.5$ and $\mathrm{MELD} \geq 30$ & $21(0.92 \%)$ & & $429(18.94 \%)$ & \\
\hline $\mathrm{Cr}>1.5$ and $\mathrm{MELD} \geq 30$ & $120(1.37 \%)$ & & $2,238(25.89 \%)$ & \\
\hline
\end{tabular}

aAdjsuted for bilirubin, INR, dialysis, patient's gender, BMI and type of transplant via logistic regression. ${ }^{\text {b} A f t e r ~ e x c l u d i n g ~ t h o s e ~ w h o ~ d i e d ~ a t ~ t r a n s-~}$ plantation. 

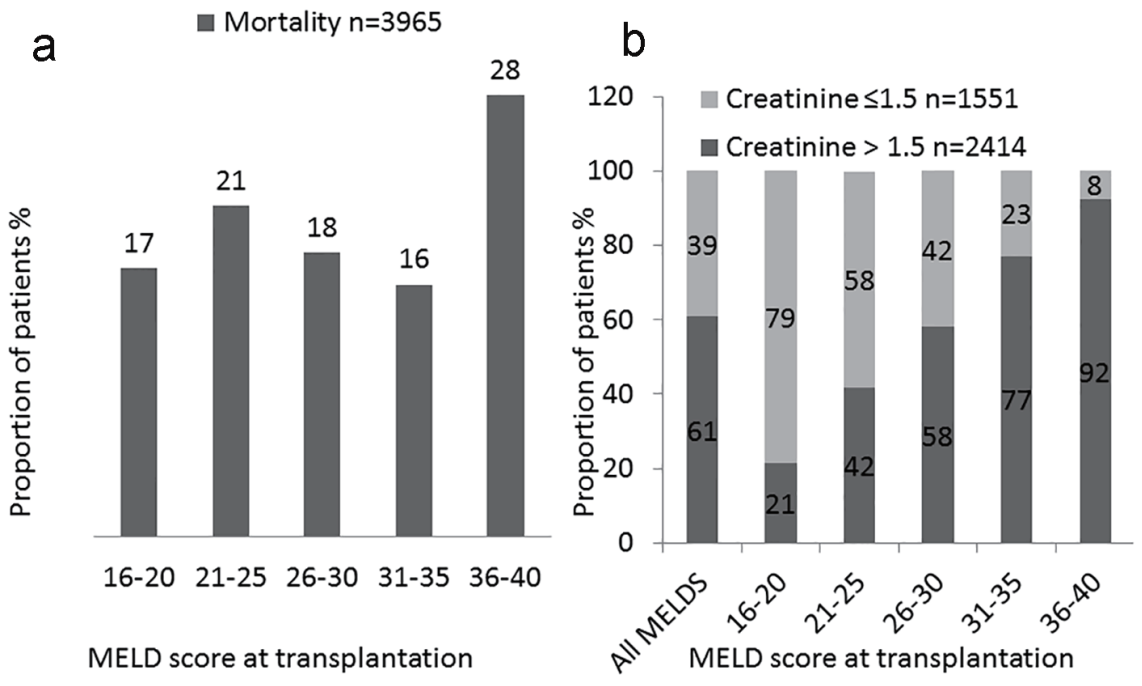

Figure 5. Analysis of deceased patients. Distribution of death within 1 year based on MELD score. (a) Proportion of post-transplantation deaths based on MELD score at transplantation in overall study cohort. (b) Proportion of post-transplantation deaths based on MELD score at transplantation in patients with and without renal dysfunctions.

less clear. One report examined survival of patients transplanted with $\mathrm{sCr}$ of $\geq 2.5 \mathrm{mg} / \mathrm{dL}$ and found overall survival and graft survival was significantly lower in those receiving LT alone vs. SLKT [16]. In a second series, similar survival rates were seen for SLKT patients compared to LT alone patients, but there was a significant improvement in graft survival among the SLKT patients [6]. In the current report, the benefits of SLKT vs. LT on survival are less clear when MELD score is considered. Survival for those with renal insufficiency was improved for those receiving SLKT when compared to patients with LT alone and with renal insufficiency in the MELD of 16 - 20 group only. For the remainder of the MELD cohorts, SLKT did not reverse the adverse effect of renal insufficiency on survival. Survival was worse for the SLKT cohorts as compared to LT alone without renal insufficiency and was similar to those with renal insufficiency who receive a LT alone (Fig. 5).

Our data however indicate that recipient survival is not necessarily improved with SLKT over other patients undergoing LT alone who have abnormal renal function. SLKT is clearly inferior compared to those without renal dysfunction, especially in those with high MELD scores (Fig. 4). Given that our data indicate no difference in survival for LT alone versus SLKT in patients with renal dysfunction, the question arises as to how many kidneys can be spared to be used for patients needing a KT. The time on dialysis is a risk factor for patient survival on the KT waiting-list making renal dysfunction in patients with liver failure an indirect risk factor for the outcome for waiting for a KT. It has been suggested previously that a more robust tool is necessary to distinguish between the two transplant groups to help reduce the number of kidney grafts used for the SLKT population [15]. Also recently, unfavorable outcomes have been reported in HCV-infected patients receiving SLK transplants [14]. As a result, the authors suggested careful donor/recipient selection when approaching HCV recipients for SLK transplants. In a study modeling the possibility of reversal of renal dysfunction in some LT patients as a result of hepatorenal syndrome, the authors noted that allocating a kidney to a patient with ESLD, who has the potential to be dialysis free without a KT, does not maximize overall outcomes when all patients are considered [13]. Furthermore, in a study using UNOS data stratified by donor subgroup, MELD score, pre- versus post-MELD era, and length of time on dialysis, the findings indicated that SLKT may be overused in the MELD era and the authors concluded that the current practice of prioritizing kidney grafts to liver failure patients results in a waste of limited resources [11].

The limitations of our study are consistent with all studies performed on data from the STAR files; the data may be incomplete and accuracy is determined by individuals compiling the data. Details that can be gleaned from a single center experience are beyond the scope of the database. Conversely multicenter data are important to understand utility of kidney grafts in LT patients. Perhaps the most important recommendation from this study is the development of better metrics to determine which patients are most likely to benefit from a SLK transplant.

In conclusion, we have shown that renal dysfunction in liver failure at the time of transplantation increases the risk for mortality of LT recipients, whether receiving a LT alone or SLKT, compared to those without renal dysfunction especially in high MELD recipients. However, low MELD patients with combined liver disease and renal insufficiency may benefit from SLKT. The differential benefit of SLKT depends upon the severity of liver disease at the time of transplantation. These findings are consistent with previous reports and support the need to improve renal function in patients awaiting LT. Recent reports have shown that improving renal function of patients with HRS-1 improves their survival irrespective of whether or not they receive a liver transplant $[22,23$, 25]. We also need to question whether MELD should be the main tool we use to allocate organs without consideration of post-transplant outcomes. Surely, the fact that $20 \%$ of patients 
with a MELD of 36 - 40 and renal insufficiency die within the first year following transplant should lead to a discussion of how we can better manage these patients. A more detailed study of this group of patients may provide insight into why they are dying and what can be done to improve their prognosis. Also, given the lack of clear survival benefit from SLK transplant, we need to better define the patients most likely to benefit from multi-organ transplant. Many of these patients are likely to have three functioning kidneys if they have AKI at the time of transplant and this is not a good use of a limited resource. Clearly defined policies are needed to better manage these very sick patients.

\section{Acknowledgments}

We appreciate the skilled secretarial assistance of Mr. L. T. Tucker and Ms. Lindsey Schader. This study was support by funds from the Liver Institute PLLC. This work was supported in part by Health Resources and Services Administration contract 234-2005-37011C. The content is the responsibility of the authors alone and does not necessarily reflect the views or policies of the Department of Health and Human Services, nor does mention of trade names, commercial products, or organizations imply endorsement by the US Government.

\section{Conflicts of Interest}

None of the authors have conflicts of interest to disclose.

\section{Author Contributions}

Shahid Habib: study idea, designing, data collection, analysis, manuscript writing, and submission. Khalid Khan: manuscript writing. Chiu-Hsieh Hsu: statistical analysis. Edward Meister: statistical analysis. Abbas Rana: study designing. Thomas Boyer: manuscript writing and critical reviewing.

\section{Abbreviations}

AKI: acute kidney injury; ANOVA: analysis of variance; ESLD: end-stage liver disease; ESRD: end-stage renal disease; HCV: hepatitis C virus; HRS: hepatorenal syndrome; INR: international normalized ratio; KT: kidney transplant; OPTN: organ procurement and transplant network; LT: liver transplantation; MELD: model for end-stage liver disease; RRT: renal replacement therapy; SLKT: simultaneous liver and kidney transplantation; STAR: standard transplant analysis and research; UNOS: United Network of Organ Sharing; sCr: serum creatinine

\section{References}

1. Organ Procurement and Transplantation Network and United Network for Organ Sharing Database [internet].
Richmond (VA): Health Resources and Services Administration, U.S Department of Health \& Human Services. 2002 - [cited 2017 Jan]. Available from: https:/optn. transplant.hrsa.gov/data/.

2. Zhu M, Li Y, Xia Q, Wang S, Qiu Y, Che M, Dai H, et al. Strong impact of acute kidney injury on survival after liver transplantation. Transplant Proc. 2010;42(9):36343638.

3. Thuluvath PJ, Guidinger MK, Fung JJ, Johnson LB, Rayhill SC, Pelletier SJ. Liver transplantation in the United States, 1999-2008. Am J Transplant. 2010;10(4 Pt 2):1003-1019.

4. Weber ML, Ibrahim HN, Lake JR. Renal dysfunction in liver transplant recipients: evaluation of the critical issues. Liver Transpl. 2012;18(11):1290-1301.

5. Sharma P, Schaubel DE, Guidinger MK, Merion RM. Effect of pretransplant serum creatinine on the survival benefit of liver transplantation. Liver Transpl. 2009;15(12):1808-1813.

6. Martin EF, Huang J, Xiang Q, Klein JP, Bajaj J, Saeian K. Recipient survival and graft survival are not diminished by simultaneous liver-kidney transplantation: an analysis of the united network for organ sharing database. Liver Transpl. 2012;18(8):914-929.

7. Nadim MK, Davis CL, Sung R, Kellum JA, Genyk YS. Simultaneous liver-kidney transplantation: a survey of US transplant centers. Am J Transplant. 2012;12(11):31193127.

8. Kliem V, Ringe B, Frei U, Pichlmayr R. Single-center experience of combined liver and kidney transplantation. Clin Transplant. 1995;9(1):39-44.

9. Moreno-Gonzalez E, Meneu-Diaz JC, Garcia G, Jimenez Romero C, Loinaz Segurola C, Gomez Sanz R, Abradelo $\mathrm{M}$, et al. Simultaneous liver-kidney transplant for combined renal and hepatic end-stage disease. Transplant Proc. 2003;35(5):1863-1865.

10. Davis CL, Feng S, Sung R, Wong F, Goodrich NP, Melton LB, Reddy KR, et al. Simultaneous liver-kidney transplantation: evaluation to decision making. Am J Transplant. 2007;7(7):1702-1709.

11. Locke JE, Warren DS, Singer AL, Segev DL, Simpkins CE, Maley WR, Montgomery RA, et al. Declining outcomes in simultaneous liver-kidney transplantation in the MELD era: ineffective usage of renal allografts. Transplantation. 2008;85(7):935-942.

12. Davis CL. Controversies in combined liver-kidney transplantation: indications and outcomes. Transplant Rev (Orlando). 2008;22(1):82-88.

13. Kiberd B, Skedgel C, Alwayn I, Peltekian K. Simultaneous liver kidney transplantation: a medical decision analysis. Transplantation. 2011;91(1):121-127.

14. Hibi T, Sageshima J, Molina E, Ciancio G, Nishida S, Chen L, Arosemena L, et al. Predisposing factors of diminished survival in simultaneous liver/kidney transplantation. Am J Transplant. 2012;12(11):2966-2973.

15. Mindikoglu AL, Raufman JP, Seliger SL, Howell CD, Magder LS. Simultaneous liver-kidney versus liver transplantation alone in patients with end-stage liver disease and kidney dysfunction not on dialysis. Transplant Proc. 
2011;43(7):2669-2677.

16. Chang Y, Gallon L, Jay C, Shetty K, Ho B, Levitsky J, Baker T, et al. Comparative effectiveness of liver transplant strategies for end-stage liver disease patients on renal replacement therapy. Liver Transpl. 2014;20(9):10341044.

17. Locke JE. To whom goes the kidney? Liver Transpl. 2014;20(9):1017-1018.

18. Fong TL, Khemichian S, Shah T, Hutchinson IV, Cho YW. Combined liver-kidney transplantation is preferable to liver transplant alone for cirrhotic patients with renal failure. Transplantation. 2012;94(4):411-416.

19. Salerno F, Gerbes A, Gines P, Wong F, Arroyo V. Diagnosis, prevention and treatment of hepatorenal syndrome in cirrhosis. Gut. 2007;56(9):1310-1318.

20. Runyon BA. Introduction to the revised American Association for the Study of Liver Diseases Practice Guideline management of adult patients with ascites due to cirrhosis 2012. Hepatology. 2013;57(4):1651-1653.

21. Kamath PS, Wiesner RH, Malinchoc M, Kremers W, Th- erneau TM, Kosberg CL, D'Amico G, et al. A model to predict survival in patients with end-stage liver disease. Hepatology. 2001;33(2):464-470.

22. Garcia-Tsao G, Parikh CR, Viola A. Acute kidney injury in cirrhosis. Hepatology. 2008;48(6):2064-2077.

23. Sanyal AJ, Boyer T, Garcia-Tsao G, Regenstein F, Rossaro L, Appenrodt B, Blei A, et al. A randomized, prospective, double-blind, placebo-controlled trial of terlipressin for type 1 hepatorenal syndrome. Gastroenterology. 2008;134(5):1360-1368.

24. Martin-Llahi M, Pepin MN, Guevara M, Diaz F, Torre A, Monescillo A, Soriano G, et al. Terlipressin and albumin vs albumin in patients with cirrhosis and hepatorenal syndrome: a randomized study. Gastroenterology. 2008;134(5):1352-1359.

25. Hanish SI, Samaniego M, Mezrich JD, Foley DP, Leverson GE, Lorentzen DF, Sollinger HW, et al. Outcomes of simultaneous liver/kidney transplants are equivalent to kidney transplant alone: a preliminary report. Transplantation. 2010;90(1):52-60. 